\title{
Zur Geschichte der Luxatio coxae congenita
}

\author{
Von M.R.Francillon
}

Im Jahre 1932 hat HaNS WüRTZ einen 2502 Fälle umfassenden Katalog von Deformitäten des Bewegungsapparates aus Geschichte, Literatur und Kunst zusammengestellt. Wir finden hier massenhaft Fuß- und Wirbelsäulendeformitäten, aber nicht mit Sicherheit eine einzige Hüftluxation. Wohl heißt es an einigen Stellen «hüftlahm» - mit diesem Ausdruck kann man aber nichts Genaueres anfangen. Diese Diskrepanz ist nicht weiter erstaunlich. Deformitäten von Wirbelsäule und Fuß fallen meist äußerlich sofort auf, während Deformitäten und Funktionsstörungen im Bereich der Hüftgelenke doch viel komplexer sind. Dazu kommt, daß die genauere Kenntnis der Hüftluxation historisch gesehen relativ jung ist, wenn sie auch früh einzelnen Autoren bekannt war; es sei hier zunächst nur auf Hippokrates, Ambroise Paré hingewiesen.

\section{Die Hüftluxation in der Geschichte}

Wir alle kennen den Chondrodystrophiker von Velasquez, den Hemiplegiker von Ribera, den Klumpfuß von Byron, den von Little und noch so manchen anderen Träger einer Deformität. Ganz anders bei der Luxation. Ich kenne aus der Geschichte nur vier Hüftluxationen, von denen die erste zu recht interessanten Kontroversen führte, da sie irrtümlicherweise einer historischen Persönlichkeit zugeschrieben wurde.

Im Juli 1935, in der Blütezeit des Braunen Reiches, wurde das, was als Gruft von Herzog Heinrich dem Löwen bezeichnet wurde, im Dom zu Braunschweig geöffnet, mit recht großem Aufwand, wie es schon die ersten Sätze der Arbeit von R. Schмidt (1936) zeigen ${ }^{1}$.

1 «Im vergangenen Juli 1935 führte das Gerücht, ein Verwahrlosungszustand mache das nötig, zur Öffnung der Gruft Heinrichs des Löwen im Braunschweiger Dom. Der Ministerpräsident des Landes Braunschweig hatte sie angeordnet. Der Führer und Reichskanzler nahm persönlich daran teil, und Sachverständige waren bei ihr gegenwärtig, insbesondere der Direktor des Kaiser-Wilhelm-Institutes für Anthropologie und Erblehre» (R.SснміDт, 1936, S. 241). - Es sollte dann eine würdige Grabstätte geschaffen werden mit einer Darstellung von Herzog Heinrichs «Lebenswerk im Osten», denn «heute sehen wir in ihm den kühnen Eroberer und weitschauenden Kolonisator weiten deutschen Lebensraumes im Osten, der vor 750 Jahren ein Werk begann, das Adolf Hitler heute vollendet» (W.Flechsig, 1939, zit. nach W.Holtzmann 1954). 
Herzog Heinrich, der als Welfe enragierte Gegner der Kaiser Friedrich I. Barbarossa und Heinrich VI., hatte 1 1/2 Jahre vor seinem Tod einen Sturz vom Pferd erlitten, der zu einer «contritio tibiae» geführt hatte ${ }^{2}$. Bei der Gruftöffnung fand man ein Skelett mit einem luxierten Hüftgelenk, das, eben aufgrund des oben angegebenen Unfalles, als das Skelett Heinrich des Löwen taxiert wurde. Aber erst siebzehn Jahre später hat dann Eugen Fischer, der frühere Direktor des Kaiser-Wilhelm-Institutes für Anthropologie und Erblehre, einen eingehenderen Bericht über die Grabfreilegung und das dabei gefundene Skelett publiziert, da ihm damals - 1935 - Schweigen auferlegt worden sei. Diese Publikation ${ }^{3}$ weckte bei dem Historiker W.Holtzmann so starke Bedenken, daß er M.Hackenbroch um eine Begutachtung der Fischerschen Deutung ersuchte. Diese Fischersche Deutung «muß» - wie Hackenbroch sagte - «den Widerspruch des Orthopäden herausfordern», und es fiel ihm nicht schwer zu zeigen, daß «noch eine ganz andere Deutung des Befundes für zulässig, ja als sehr viel wahrscheinlicher gehalten werden» könne (Hackenвroch, S.489 und 491). Er führt aus, daß alle von Fischer erhobenen Befunde (Luxation, Form der Pfanne, Schenkelhalsverkürzung, abnorme Torsion, Verkleinerung des GCD-Winkels, Atrophie von Femur, Patella und Tibia) unmöglich auf einen $1 \% \frac{1}{2}$ Jahre früher durchgemachten Unfall zurückgeführt werden könnten. «Der Skelettbefund kann nicht als traumatisch hervorgerufen angesehen werden. Er ist vielmehr das Ergebnis einer sehr früh einsetzenden Entwicklungsstörung am Hüftgelenk, vielleicht schon der Hüftgelenksanlage, wahrscheinlich die Folge einer sogenannten angeborenen Verrenkung»

2 «dux de equo corruit et ex contritione tibiae ab itinere, quod coeperat, impeditus est» (Steterburger Chronik von Probst Gerhard II in Mon.Germ.Hist., S.16, 227; zit. nach W. HoltzmanN, 1954).

3 Aus Fischers Arbeit sie zitiert: Pfanne dreieckig als «querovale Mulde». "In dieser Pfanne hatte ein Oberschenkelkopf überhaupt nicht mehr Platz» ... Linke Beckenhälfte kleiner als rechts ... Beckenhöhe links um $8 \mathrm{~mm}$ kürzer als rechts ... Femurkopf und -hals schwer verändert», Kopf «pilzförmig », Kopfdurchmesser li 33 mm, rechts 45 mm; Hals um Hälfte verkürzt; Oberschenkel li um $7 \mathrm{~mm}$ kürzer; Patella li um 2,5 mm kürzer und um $4 \mathrm{~mm}$ schmäler; auch Tibia li dünner als re. «Dieser ganze Befund zeigt deutlich die Folgen einer schweren Verletzung der linken Hüfte» ... «Alle diese Dinge ... beweisen damit einwandfrei, daß die Gebeine die des Sachsenherzoges sind und keine anderen ... Die Deutung dieses Befundes dürfte einwandfrei die sein, daß der Schenkelhals gebrochen war, der Bruch bis an den Kopf heranging und der Hals dann in den Kopf eingerammt war. Gleichzeitig war der Oberschenkel aus der Gelenkpfanne herausgewichen (luxiert)» (E. Fischer, 1952). 
(HACKenbroch, S.494/495). Auch aus anderen historischen Gründen (W.Holtzmann) mußte abgelehnt werden, daß es sich bei diesen Gebeinen um die des großen Sachsenherzogs handelte. Später hat dann Fischer seine Deutung ganz erheblich eingeschränkt und gab zu, daß die traumatische Genese dieser Skelettveränderungen nicht beweisbar sei, wie er es 1952 behauptet hatte, sondern daß die Befunde mehr «für eine traumatische als für eine angeborene Luxation» sprächen und daß eine «wirklich einwandfreie Entscheidung unmöglich» sei, wobei aber doch bemerkt werden darf, daß seine Einwände nicht überzeugend sind. Wie dem auch sei, wir dürfen uns der Hackenbrochschen Deutung anschließen und können lediglich sagen, daß wir es hier mit einem Skelett aus dem Mittelalter zu tun haben, das fast alle Zeichen einer nicht reponierten kongenitalen Hüftluxation zeigt, und daß es sich höchstwahrscheinlich nicht um das Skelett von Heinrich dem Löwen ${ }^{4}$ handelt.

Die drei nächsten Luxationen führen uns in die Übergangszeit vom 15. zum 16. Jahrhundert, also zum Übergang von Mittelalter zu Neuzeit. Es handelt sich um Anne de Bretagne und ihre zwei Töchter Claude und Renée. Ein strikter Beweis für das Vorliegen einer Luxatio coxae cong. läßt sich hier nicht führen, da während der Französischen Revolution die Grabstätten in der Kathedrale von St-Denis und Nantes zerstört und die Skelette zerstreut wurden. Herkunft - die Bretagne als Luxationsgegend ${ }^{5}$ und Heredität sprechen aber doch eine so deutliche Sprache, daß, um die schöne Formel zu brauchen, wir hier mit an Sicherheit grenzender Wahrscheinlichkeit kongenitale Hüftluxationen annehmen müssen.

${ }^{4}$ Ganz abgesehen davon, daß in den reichlich vorliegenden historischen Dokumenten nirgends die Rede davon ist, daß Heinrich der Löwe je gehunken habe (W. Holtzmann). Auch mit dem Hinweis, den Frscher 1956 bringt, auf Anregung von L. Kreuz hin, daß einseitige Luxationen nicht immer von ihrem Leiden behindert seien, ist nicht viel anzufangen. Dasselbe gilt von dem von Fischer zitierten Satz von L. KreUz «Der Träger einer einseitigen Luxation, der wie die Ritter und Könige seinerzeit viel zu Pferde saß und sich in schweren Rüstungen bewegte (die geradezu die Vorläufer unserer Schienenhülsenapparate sind!) braucht in keiner Weise offenkundig von dieser Luxation behindert gewesen zu sein», wobei Fischer selbst im Hinblick auf das Alter des Sachsenherzogs diese Möglichkeit bezweifelt (Fischer, 1956).

5 Die Bretagne ist schon lange als «Luxationsgegend» bekannt. So gibt LE Davavy für den Südwesten der Bretagne eine Luxationsfrequenz von $5 \%$ der Bevölkerung, an und es ist nicht ohne Grund, daß Leveuf und BerTrand ihr Luxationsbehandlungszentrum gerade in der Bretagne, in Pont-l'Abbé, einrichteten. 
Die Geschichte von Anne de Bretagne, wohl der bedeutendsten Königin Frankreichs, ist interessant und typisch für die ganze Politik, speziell für die Heiratspolitik ihrer Zeit. «La belle boiteuse», wie sie auch genannt wurde $^{6}$, war, als ihr Vater ohne einen Sohn zu hinterlassen 1488 starb, Erbin und Herzogin der Bretagne. König Maximilian, der spätere Kaiser, war durch seine Frau Maria von Burgund, der 1482 verstorbenen Tochter Karls des Kühnen, Herrscher des Gebiets, das jetzt Luxemburg, die Franche Comté, Belgien und die Niederlande umfaßt. Die habsburgische Heiratspolitik zeigte sich dann auch darin, da 3 er sich - allerdings nur per procurationem; er hat Anne nie gesehen - 1490 mit Anne verheiratete ${ }^{7}$. Das bedeutete Einkreisung Frankreichs. Es kam zum Krieg, und schließlich, nach langen Verhandlungen und da Kaiser Maximilian nicht zu Hilfe kommen konnte (wollte ?), entschloß sich Anne zur Heirat mit König Charles VIII., um ihrem Herzogtum weitere Zerstörungen zu ersparen (1491). Als Charles VIII. 1498 ohne Nachkommen - seine vier Kinder von Anne waren alle jung gestorben - starb, wurde sein Neffe Louis XII. sein Nachfolger, der dann Anne, seine alte Liebe, 1499 heiratete.

Was Anne als Königin für Frankreich bedeutete, gehört nicht hierher ${ }^{8}$. Auch ihre beiden Töchter hinkten (ausdrücklich heißt es «pareillement disgraciées de la nature» wie Anne und «Comme Anne Claude était boiteuse»); die älteste, Claude, wurde Gemahlin von König François I ${ }^{\text {er }}$, dem Gegen-

${ }^{6}$ «La reine est petite; elle est aussi maigre de sa personne, boiteuse d'un pied, et d'une façon sensible, bien qu'elle s'aide de chaussures à talon élevé, brunette et fort jolie de visage...» (Bericht des venetianischen Gesandten Z.Contarini, zit. nach E.GABory, 1941).

7 Diese Heirat war schließlich nichts anderes als Ausdruck des Antagonismus zwischen dem Deutschen Reich bzw. Habsburg und dem französischen Königtum. Es sei u. a. nur erwähnt, daß, etwa 300 Jahre früher, Kaiser Otto IV., der Sohn des oben genannten Heinrich des Löwen, 1214 von König Philippe Auguste von Frankreich bei Bouvines geschlagen worden war. Dieser Antagonismus zwischen dem Reich und Frankreich bzw. Habsburg und Valois wird um die Wende des 15. zum 16. Jahrhundert noch dadurch gesteigert, daß König Charles VIII. seine Verlobung mit Margareta, der Tochter Kaiser Maximilians aus erster Ehe mit Maria von Burgund und späterer Statthalterin in Brüssel, brach und sie ihrem Vater zurückschickte, eben um Anne de Bretagne zu heiraten - also doppelter Affront gegen Maximilian.

8 "Anne de Bretagne est de toutes les reines de France celle qui demeure la plus populaire»... «Aucune reine ne voyagea plus qu'Anne...» (GABORY). Anregungen für Architekten (Umbau der Schlösser von Amboise und Blois) und Dichter; vor allem ist hier auf das Livre d'Heures d'Anne de Bretagne hinzuweisen, siehe u. a. Paul Lacrorx. 
spieler von Kaiser Karl V., und die jüngere, Renée, die Anne charakterlich sehr ähnelte, wurde Herzogin von Ferrara, wo sie u.a. Clément Marot und Calvin empfing ${ }^{9}$.

\section{Zur Geschichte der Kenntnis der Hüftluxation}

Wissen wir somit recht wenig über die Hüftluxation bei historischen Persönlichkeiten, so weiß man doch wesentlich mehr über die Entwicklung unserer Kenntnisse dieses Leidens. Diese Entwicklung war, wie wir sehen werden, manchmal mit recht unschönen und kleinlichen Polemiken verbunden - die invidia ist als Charistericum humanum auch bei Medizinern gut ausgebildet...

Ob im Altertum in Ägypten die Luxation bekannt war, wissen wir nicht. Fünf Fälle von angeblich angeborener Hüftgelenksluxation sind, wie P.Hengen schreibt, mit Vorsicht aufzufassen; eher handle es sich, wie er mir persönlich mitteilte, um Epiphysenlösungen.

Mit Sicherheit dagegen geht unsere Kenntnis der Luxation auf HippoKRATES bzw. die hippokratischen Schriften zurück. An drei Stellen des Buches über die Luxationen ${ }^{10}$ wird die kongenitale Hüftluxation erwähnt bei der Schilderung des Hüfthinkens, wobei er im klinischen Bild aus naheliegenden Gründen nicht zwischen angeborenen, traumatischen oder coxitischen Luxationen scheidet; an zwei Stellen (Kapitel 52 und 54) heißt es aber, daß «diejenigen, die diese Luxation schon im Mutterleibe erleiden

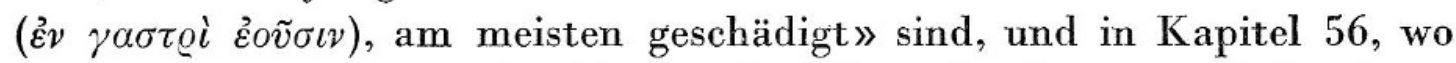
sich eine ausgezeichnete Schilderung der bilateralen Luxation findet, kann

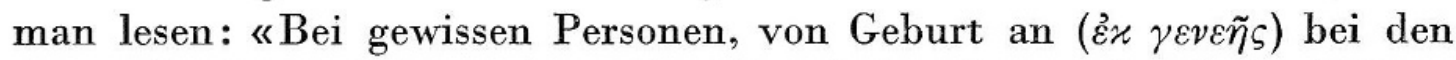
einen, durch die Folgen einer Krankheit bei anderen, sind beide Hüften nach außen luxiert.» ${ }^{11}$ Die Darstellung der Hüftaffektionen ist in ihren Details hochinteressant. «Das Studium des Hippokrates ist außerordent-

9 Ausführlich über Renée de France siehe V. ChLEDowsKi.

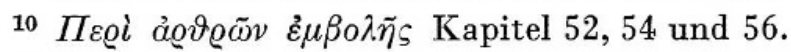

11 In diesem Zusammenhang sei erwähnt, daß die Deutung der in Kapitel 53 befindlichen Erzählung, die auch LoRENZ als Beleg für Hippokrates' Kenntnis der angeborenen Luxation zitiert, nach welcher die Amazonen den Kindern männlichen Geschlechts die Hüften luxieren, wobei von innerer und äußerer Luxation die Rede ist, nach P.BADE auf einer ungenauen Interpretation bzw. Überzetzung beruht. - Über Hippokrates und die Luxatio coxae cong. bringt auch GURLT recht eingehende Angaben. 
lich lehrreich und beweist uns die glänzende Beobachtungsgabe dieses griechischen Arztes. Bis in das späteste Mittelalter haben wir jedenfalls keine bessere Beschreibung über Erkrankungen der Hüfte erhalten» (P.BADE, S.41).

Daß aber immer wieder kongenitale Hüftluxationen vorkamen, läßt sich aus Gräberfunden belegen. So hat CaLvin WeLLS ein Skelett aus der spätangelsächsischen Periode, also aus dem 9. oder 10.Jahrhundert n.Chr., publiziert, das einer etwa fünfzigjährigen Frau gehörte, das links eine typische kongenitale Hüftluxation aufwies.

In der Tat muß man noch weitergehen. Erst in der Neuzeit bei Ambroise Paré (1517-1590) findet sich Neues über die Hüftluxation in dem Hinweis, daß die Einrenkung nicht möglich sei, weil die Pfanne zu flach sei.

Wir müssen aber bis in die zweite Hälfte des 18. Jahrhunderts gehen, um Genaueres über die Hüftluxation zu erfahren. Auch N.ANDrys' Beschreibung, in der er das typische Hinken beschreibt ${ }^{12}$, erreicht bei weitem nicht die Klarheit und Lebhaftigkeit von Hippokrates.

Genauere Angaben über Klinik und Pathologische Anatomie finden sich erst 1783 bei Giovanni Batrista Paletta, dem das Verdienst zukommt, der erste genaue Beschreiber dieses Leidens in der Neuzeit zu sein ${ }^{13}$.

Palettas Arbeiten von 1783 und 1820 waren Guillaume Dupuytren (1777-1835) wohl unbekannt, als er 1826 die Entdeckung einer neuen bisher unbekannten Krankheit beschrieb: «Il est une espèce de déplacement de l'extrémité supérieure du fémur, de laquelle je n'ai trouvé aucune indication dans les auteurs, quelques recherches que j'ai faites pour la découvrir.» Gleichzeitig aber warnte er vor jeglicher Therapie, indem er sagte: «On se consolerait aisément de ne pas connaître la cause de ces déplacements si l'on connaissait les moyens de les faire cesser ou du moins de pallier leurs mauvais effets; malheureusement il n'en est pas ainsi et ces déplacements ne comportent ni remède curatif ni même de palliatif bien efficace. » 14

12 «dandinant» (L'Orthopédie, I, 304, Paris 1741).

13 Adversaria chirurgica prima Milano 1783. Kapitel: «De claudicatione congenita». Hier bringt er die Anatomie einer Lux. bilat.: «orificium ipsum cotylis fere ovale... Ab acetabuli centro usque ad marginem segmenti inferiores surgebat tuber, aut colliculus osseus... Femur, pene dixerim, acephalum fuit... » Im ganzen bringt er die Pathol. Anatomie von drei Hüftluxationen.

14 Dupuytren, Mémoire sur un déplacement originel ou congénital de la tête des fémurs, (Rep.gen.Anat., Physiol.etc., 1826 Acad.Méd.Paris). 
DupuYtren genoß eine ungeheure Autorität, war er doch auf dem Festland der größte Chirurg seiner Zeit ${ }^{15}$. Dieses Verdikt, das er auch noch 1835 im Jahre seines Todes aussprach in seinen Leçons orales de Clinique chirurgicale, hat sicher manchen abgehalten, sich mit dieser «neuen » Krankheit zu befassen, und zeigt wieder einmal, wie gefährlich Autorität in medizinischen Dingen sein kann. Auf der anderen Seite aber hat Dupuytren gerade durch seine «Entdeckung» die allgemeine Aufmerksamkeit auf dieses Leiden gelenkt ${ }^{16}$.

Es darf aber beigefügt werden, daß die genauere Kenntnis der Anatomie dieses Leidens in einem gerichtlich-medizinischen Fall DupuYTREN der Gerichtsbehörde die Möglichkeit gab, den Toten zu identifizieren und dann den Mörder zu ermitteln (VaLENTiN, Geschichte der Orthopädie).

Diese negative Einstellung DuPUYTRENs ist aber auch wieder begreiflich, wenn man an die diagnostischen Schwierigkeiten (auch hinsichtlich des Behandlungsresultates), dieser Vorröntgenära denkt. So wurden, wenn jemand auf diesem Gebiet in Frankreich Ergebnisse mitteilte, immer wieder Ärztekommissionen zur Kontrolle eingesetzt.

Eigenartig mag erscheinen, daß der bedeutendste und gleich alte Zeitgenosse Dupuytrens, Jacques-Mathieu Delpech (1777-1832), in seiner Orthomorphie (1828, deutsch 1830) kaum etwas Eigenes über die Hüftluxation sagt, obschon er PALETta ausgiebig zitiert. Diese Zitierungen bringt er aber wohl vor allem, um zu zeigen, daß Dupuytren Palettas Text nicht gekannt hat. Wir dürfen auch hierin einen Beleg dafür sehen, daß diese beiden großen Chirurgen, der eine in Paris, der andere in Montpellier, nicht gut aufeinander zu sprechen waren.

Aber trotz des Einflusses und des Verdikts Dupuytrens - oder vielleicht gerade deshalb! - ließ das Problem die Ärzte nicht mehr los. So versuchte François Humbert (1776-1850) mit gewaltsamer Extension eine Reposition zu erreichen; bei diesen Transformationen, wie sie Pravaz bezeichnete, hat es sich aber wohl bestenfalls nur um Inversionen oder um Trans-

15 «Dupuytren a été le plus grand chirurgien de son temps» (Mondor, S.320).

16 Pravaz sagt dazu: «On a dit qu'il n'y avait de nouveau que ce qui avait été oublié; sous ce point de vue du moins, DUPUYTREn a bien réellement découvert une malformation nouvelle. Son immense renommée, le retentissement de sa parole au milieu de la première société savante de l'Europe ont en effet remis en lumière une question dont l'importance était méconnue, et qui était restée jusqu'alors ensevelie, pour ainsi dire, dans la poussière des bibliothèques» (Pravaz, 1847, S.10). 
positionen in das Foramen obturatum gehandelt; es waren Verbesserungen, also Fortschritte, die auch Pravaz anerkannte, aber für sichere Repositionen ließen sich keine Anhaltspunkte finden.

Schon neun Jahre später, 1834, wurde Dupuytrens Pessimismus durch Charles-Gabriel Pravaz (1791-1853) widerlegt - ein weiterer Beweis, daß solche Prognosen in der Medizin glücklicherweise eine sehr zweifelhafte Sache sind ${ }^{17}$.

«Pravaz gebührt unstreitig das Verdienst, als erster wirkliche Repositionen zustande gebracht zu haben...» und «das wurde noch in unseren Tagen von CALOT bestätigt, der ein kleines Kind mit Luxation behandelte, dessen Großmutter von Pravaz geheilt worden war (Lorenz, 1920, S. 7 und 8).

Aber erst dreizehn Jahre später, 1847, veröffentlichte PRAvaz sein Traité théorique et pratique des Luxations congénitales du Fémur, ein Buch, «dessen Lektüre» - wie VALENTIN mit Recht sagt - «heute noch warm zu empfehlen und ein wahrer Genuß ist.»

Pravaz geht auf Palettas anatomische Untersuchungen zurück, die ihm bewiesen, daß eine Reposition möglich sei unter folgender Voraussetzung: «commencer aussi tôt que possible le traitement des luxations congénitales de la hanche» - also ein sehr modernes Prinzip. War bisher (Humbert, Jalade-Lafond u.a.) nur eine axiale Extension angewandt worden (und zwar ergebnislos punkto Reposition), so ging Pravaz von einem anderen Prinzip aus: zunächst langsame monatelange leicht schräge Extension mit Gegenzügen an Axilla und Tuber ischii, dann, wenn der Femurkopf in Pfannenhöhe war, Abduktion der Beine unter Pelottendruck am Femur. Auch diese zweite Etappe wurde öfters wiederholt, bis Pravaz sich von der sicheren Reposition überzeugt hatte; dann für Monate ohne Belastung Übungsbehandlung in einem «char à engrenage et à bielles» und dann Gehstuhl und Krücken für Gehübungen.

125 Seiten seiner Monographie sind der Kasuistik gewidmet. Es handelt sich um 19 Patienten, deren Beschreibung so ausführlich, so genau ist, daß man 5 Fälle ausscheiden kann (2 septische Luxationen, 1 Perthes, 1 bilate-

17 Bei der ersten Reposition, die Pravaz in Paris bei einem achtjährigen Mädchen 1834. durchführte, kam es allerdings einige Monate später durch Sturz zu einer Reluxation. Die Patientin kam nicht mehr zu Pravaz, der nunmehr in Lyon tätig war. Von anderer Seite dann durchgeführte Repositionsversuche führten nicht zum Ziel, da sie, wie er sagte, nicht nach seiner Methode durchgeführt wurden. 
rale Luxation im Alter von 19 Jahren, die Pravaz selbst wegen des Alters als "pronostic défavorable» ausschließt und 1 rechtsseitige Luxation im Alter von 15 Jahren, bei der wegen mangelnder Geduld die Behandlung nach $1 \mathrm{Jahr}$ abgebrochen wurde), so daß noch 14 Patienten im Alter von $2 \frac{1}{2}$ bis 15 Jahren bleiben. In 10 Fällen ist in der Tat die Reposition gelungen (11 Hüften). Die meisten Patienten wurden vor und nach der Behandlung von Ärzten bzw. Ärztekommissionen untersucht - dies als Hinweis auf die kritische Einstellung von Pravaz selbst ${ }^{18}$.

Wie bewunderungswürdig genau Pravaz untersuchte geht aus folgendem Beispiel hervor:

o 6 jährig. Lux. coxae cong. sin. Kontrolle durch ärztliche Kommission. Extension 4 Monate lang, dann Reposition und Übungsbehandlung. Bei der Kontrolle durch die Kommission 3 Jahre später: Reposition festgestellt. Die Genauigkeit der Untersuchung vor der Therapie wird durch folgende Bemerkung bestätigt: «le col du fémur s'inserait au corps de cet os en un point de sa circonférence situé plus antérieurement que d'ordinaire» - also Feststellung einer vermehrten Antetorsion!

Einen Nachteil hatte die Behandlung: ihre lange Dauer ${ }^{19}$. Das galt auch für jüngere Patienten ${ }^{20}$. Wurde seine Methode auch verschiedentlich in Deutschland, Frankreich, England, den Vereinigten Staaten von Einzelnen mit Erfolg angewandt (Zusammenstellung bei B. VALENTIN), so geriet sie doch bald in Vergessenheit, und zwar doch wohl wegen ihrer Langwierigkeit.

18 Einige Bemerkungen Pravaz' erscheinen mir so wichtig, daß ich sie hier folgen lasse: S. 118: «L'anatomie pathologique des déplacements originels du fémur n'implique point, comme le pensait Dupuytren et comme le pensent encore quelques chirurgiens prévenus, l'impossibilité de réduire ce genre de luxations.» S.119: «Les observations anatomiques ayant ètabli que l'étendue des changements, apportées à l'état normal des parties de l'articulation coxo-fémorale et de ses annexes, était proportionnelle à l'ancienneté de la luxation, c'est-à-dire à l'âge des sujets, le pronostic est d'autant plus favorable que cet âge est moins avancé.»

19 Als Beispiel sei zitiert: Fall 6.1838.13 J. o Lux.bilat.Vor Therapie Kontrolle durch Ärztekommission. Extension 19 Monate der einen Seite, dann Reposition durch Abduktion. 1 Monat später Extension der anderen Seite 6 Monate lang, dann Reposition. Dann 1 Jahr Übungsbehandlung. Ärztekontrolle nachher: «...reconnurent la réalité de la réduction à la disparition de tous les symptômes qu'ils avaient notés lors de leur premier examen.»

${ }^{20}$ Die jüngste Patientin war 23/4 Jahre alt (Lux.sin.). Extension 2 Monate, Reposition durch Abduktion. Resultat gut. In der Epikrise spricht Pravaz von der «grande jeunesse du sujet qui a rendu la réduction plus facile». 
Sein Name fiel aber doch nicht in Vergessenheit: er lebt weiter als Erfinder der Injektionsspritze ${ }^{21}$.

Dazu kam die Zunahme pathologisch-anatomischer Kenntnisse dieses Leidens und die Entwicklung der Chirurgie, so daß, wie Valentin schreibt, für die Jahre von 1860 bis 1890 nur wenig Autoren zu finden sind, die sich mit der sogenannten unblutigen Therapie dieses Leidens befaßt haben.

In diesem Zusammenhang muß aber doch BucKMinster Brown (1819 bis 1891) erwähnt werden, der, bei Little, Guérin, Bouvier und Stromeyer ausgebildet, der erste war, der sich in den USA ganz der Orthopädie widmete und der der erste war, der in den USA eine Hüftgelenksluxation reponierte, und zwar mit der Pravazschen Methode. (4 jährig, o , bilaterale Luxation; 2 Jahre lang Extension!) ${ }^{22}$

So kam es zu einer Periode, in welcher versucht wurde, auf operativem Wege eine Besserung, ja eine Heilung zu erzielen.

Zuvor sei aber auf einen aus dieser Zeit stammenden Vorschlag hingewiesen, der leider erst viele Jahrzehnte später, ja fast ein halbes Jahrhundert später, seine Realisation fand.

Am 8. Kongreß der Deutschen Gesellschaft für Chirurgie verlangte 1879 W.Roser, dem man u.a. die Roser(1846)-Nélaton(1847)-Linie verdankt, «daß man die Kinder nicht, wie bisher, 2 Jahre alt werden lasse, ohne ihre Hüftverrenkung zu diagnostizieren», denn: «Die frühzeitige Diagnose solcher Verrenkungen ist aber gewiß die erste Bedingung ihrer Heilbarkeit. Ich glaube, daß viele dieser Fälle, sogar die meisten derselben noch heilbar wären, wenn man die Krankheit an den Neugeborenen erkennen und wenn man sogleich die nöthigen Abductionsverbände anlegen würde». Er schlug vor, den Säuglingen Gipsstiefelchen anzulegen und die Beine durch einen angegipsten Querstab gespreizt zu halten. «Ein Keilpolster, mit Gummizeug überzogen, könnte noch hinzugefügt werden, vielleicht würde es sogar allein schon zur Erfüllung des Zweckes dienen.»

Mit diesem Vorschlag, den er selbst nicht in die Tat umsetzte, war Roser seiner Zeit weit voraus, wenn auch einige, wie z. B. Max Schede, Mikulicz, sich der Abduktionsmethode bedienten. Bis aber Frühdiagnose und Frühbehandlung sich durchsetzen konnten und Allgemeingut wurden, mußten noch manche Dezennien vergehen.

${ }^{21}$ Konstruktion einer Metallspritze und einer Hohlnadel, die er benutzte zur Injektion von Eisenchlorid in Aneurysmen zur Blutkoagulation.

22 Über B. Brown siehe u. a. NICHoLson. 
Noch 1864 konnte Stromeyer in seinem Handbuch der Chirurgie schreiben: «Angeborene Luxationen des Hüftgelenkes geben bis jetzt wenig Aussicht auf Heilung» (Band II, S. 975).

Interessant ist, daß von den operativen Methoden die Pfannendachplastik zu den ältesten gehört.

So suchte J. Guérin (1801-1866), den Stromeyer als den «Heißsporn der operativen Orthopädik» bezeichnete, durch subkutane Skarifikationen das Periost zur Pfannendachbildung anzuregen; von einem Erfolg ist nichts bekannt.

Die erste eigentliche Pfannendachplastik stammt von Franz KönIG (nachdem MARgary, allerdings unter partieller Kopfresektion vergeblich ähnliches versucht hatte); KöNIG bildete aus dem Ilium einen KnochenPeriost-Lappen, den er über den Kopf hinunterklappte. Vier Wochen später starb die Patientin an Diphtherie. Bei der Sektion zeigte sich der «Befund eines knöchernen Firstes, welcher als Ersatz der hinteren Pfannenwand durch Ablösung des Knochenlappens geschaffen wurde»(Lorenz, 1895, S. 191).

MAx Bonst, der spätere Pathologe in München, hat in seiner Würzburger Dissertation 1892 einen von ScHönborN so durchgeführten Eingriff geschildert (o 18 J., Lux. bilat.; Op. links: Periostknochenlappens fächerförmig bis zur Kapselinsertion herabgeschlagen). Das Ergebnis wurde als sehr gut taxiert, und in der Tat sieht man auf den postoperativen Photographien der Dissertation, daß das Trendelenburgsche Phänomen links negativ war, während es auf der rechten, nicht operierten Seite noch positiv war.

In der gleichen Periode mehrten sich die Versuche, auf operativem Wege eine Reposition zu erreichen. Nach einem vergeblichen Versuch MARGarys ${ }^{23}$ (der fünfzehnjährige Patient starb 11 Tage post op. an Pyämie) gelang es A. Poggi 1888 erstmals, den Kopf in die am normalen Pfannenort angelegte, künstlich vertiefte bzw. gebildete Pfanne zu reponieren; Kontrolle nach 13 Monaten: gut, Gelenk frei beweglich, Hinken fast verschwunden ${ }^{24}$. Dann haben im deutschen Sprachgebiet A. Hoffa (seit 1890), A. Lorenz (seit 1892), E. Kirmisson in Paris (dieser aber mit wenig Erfolg,

23 Über MARgary, mit Panzieri 1884 Begründer des Archivio di Ortopedia, siehe V.Putti, 1938.

24 «Vi è rimasto solo un insignificante grado di zoppicamento che potrebbe corregersi con una scarpa adatta» (Arch.ortop.VII, 105, 1888 zit.nach LoRENz 1895). 
da er die neue Pfanne ganz durchbohrte) die offene operative Reposition weiter ausgebaut. So konnte HoffA, der damals noch in Würzburg tätig war, am Internationalen Kongreß in Rom 1894 berichten, daß er diese offene Reposition bisher 75mal durchgeführt habe und daß er «mit den Resultaten sehr zufrieden" sei. A. Lorenz hat dann 1892 zunächst die Hoffasche Methode angewandt; sein erster Fall verlief aber so unerfreulich (Reluxation, monatelange Eiterung), daß er dann im Gegensatz zu HofFA auf dessen ausgiebige Muskeldurchtrennung verzichtete außer dem «funktionell bedeutungslosen Tensor fasciae latae» (Lorenz, 1895, S. 359) und dafür eine prä- und intraoperative starke Extension durchführte ${ }^{25}$. So konnte er auf dem oben genannten Kongreß auf 63 auf diese Weise von ihm operierten Hüftgelenke verweisen.

Waren die Jahre 1838-1847 für die Luxation insofern bedeutsam, daß in dieser Zeit Pravaz erstmals in geeigneten Fällen ihre Heilbarkeit demonstrieren konnte, so waren die Jahre 1888-1894 noch bedeutsamer, nicht nur in der Umkehr von der operativen zur konservativen Therapie, sondern weil sie ein ganz neues Prinzip brachten, das sich für die nächsten Dezennien durchsetzte: hierin liegt die große Bedeutung des so bald von vielen vergessenen Agostino Paci aus Pisa (1845-1902).

Entscheidend war in dieser Hinsicht der 11. Internationale Medizinkongreß, der vom 29.3. bis 5.4.1894 in Rom stattfand und an dem GHiLlini, Hoffa, Kirmisson, Lorenz, Mikulicz und Pagi über die Therapie der Luxation sprachen, wobei letzterer erstmals vor einem internationalen Forum über seine Behandlungsmethode sprach, die er 1886 erstmals demonstriert und 1888 publiziert hatte.

PACI erdachte, wie LoRenz 1895 selbst schreibt, «mit unbestreitbarem Scharfsinne ein Repositionsverfahren, welches im Gegensatz zur Extension gewissermaßen durch Circumduction des Schenkelhalses den Kopf an die richtige Stelle zu verlagern sucht» (S. 155).

${ }^{25}$ Seine ersten zwölf offen in der Chirurgischen Universitätsklinik Wien (Prof.E.Albert) durchgeführten Repositionen waren aber im Ergebnis so katastrophal (dreimal Eiterung, zwei Todesfälle an Sepsis), daß er die Klinik verließ («denn die bisherigen Mißerfolge waren ja in erster Linie durch die minder günstigen aseptischen Verhältnisse einer großen Klinik bedingt», Lonenz, 1895, S. 296) und seine Arbeit dann in einer Privatheilanstalt fortsetzte, mit so günstigem Resultat, daß er 1895 einen Bericht über hundert glatt verlaufener Fälle im Alter von 2 bis 19 Jahren innerhalb von zwei Jahren veröffentlichen konnte. 
Pacis 4 Tempi sind folgende: In Narkose:

1. Flexion von Hüfte und Knie

2. Leichte Abduktion des Oberschenkels

3. Außenrotation

4. Streckung im Hüftgelenk, manchmal in Abduktion. Dann Gipsfixation für 4 Monate.

In der gleichen Sitzung berichtete er über 23 eigene Fälle und über etwas mehr als 40 Fälle, die von anderen Kollegen, meist in Italien, nach seiner Methode behandelt worden waren.

Entscheidend war aber wohl vor allem, daß PACI an diesem Kongreß das Beckenpräparat eines siebenjährigen Mädchens mit bilateraler kong. Hüftluxation zeigen konnte, das 4 Monate nach der Reposition an Dysentrie verstarb ${ }^{26}$. Beide Hüftgelenke waren reponiert ${ }^{27}$.

PACIS Ausführungen und vor allem seine Demonstration müssen einen ungeheuren Eindruck gemacht haben ${ }^{28}$ : am 5. 4. 1894 ging der Kongreß zu Ende, und bereits seit dem 10. 4. 1894 hat LoRenz, wie er 1895 schreibt, in jedem ihm «geeignet scheinenden Falle die 4. Tempi möglichst genau nach dem mir auch aus eigener Anschauung bekannten Verfahren PAcis ausgeführt» - aber mit negativem Resultat, wie ihm jedesmal nachher der Ein-

${ }^{26}$ Reposition durch A. NotA im Ospedale Regina Margherita in Torino 1892, also durch einen Vorgänger von Ugo CAMERA.

27 Es ist wahrlich hochinteressant zu lesen, was A. Lonenz 1895 hierzu schrieb: «Ich habe das interessante Präparat in Rom gesehen ... Es fand sich beiderseits eine genau den Dimensionen des Schenkelkopfes entsprechende neugebildete (sic!) Pfanne, deren Localisation namentlich links dem normalen Pfannenorte genau entsprach, während sie rechts etwas weiter außen zu liegen schien... Ich kann nicht verschweigen, daß ich bis zu diesem Augenblicke mich eines großen Mißtrauens gegenüber der PAcischen Methode nicht entschlagen konnte... Nunmehr aber muß ich gestehen, daß ich von der Leistungsfähigkeit der PACIschen Methode im Sinne der weiter unten darzulegenden Einschränkungen vollkommen überzeugt bin ...» (S.160). Er war so überzeugt, daß er in seiner Monographie von 1895 sogar eine Abbildung des PAcischen Präparates brachte!

${ }^{28}$ Zur Demonstration, die PACI nach seinem Vortrag durchführte (Demonstration seiner Methode und des Präparats), schreibt A. R. Jones sehr anschaulich: «It was a thrilling and dramatic moment. From that time manipulation held the field and open operation was reserved for the exceptional case. None was more impressed than Lorenz. He gave up his own operation which he had advocated befor the Congress and the following year brought out a modification of Paci's procedure.» Und Bick sagt: "Agostino PACI, whose name, unfortunately, has never been adequately appreciated outside of his own country, was one of the outstanding orthopedists of 19th century Italy» $(194.8$, S. 377). 
griff zeigte, der zur offenen Reposition führte. Er kam daher zum Schluß, daß es sich bei den PAcischen Fällen bestenfalls um Transpositionen handle, und zwar meist in Form einer vorderen Luxation. Zweifellos hat PACI gerade bei älteren Kindern mit seiner Methode lediglich Transpositionen erreicht; daß er aber in der Tat Repositionen erreicht hat, beweist das von ihm demonstrierte Präparat, ganz abgesehen von seinen und seiner Nachahmer klinischen Erfolgen. Auch hier kommt alles auf das Alter des Patienten an!

Noch in seiner 1895 erschienenen Monographie, in der er auch die PACIsche Methode bespricht, zeigt sich LoREnZ als absoluter Anhänger der offenen Reposition.

Aber nicht für lange Zeit. Im gleichen Jahr (1895) arbeitete er seine Methode aus, indem er Reposition und Retention scharf trennte, d.h. indem er die PAcische Flexions-Abduktions-Stellung noch steigerte und in dieser Stellung dann für mehr oder weniger lange Wochen fixierte. Lorenz' Verdienst liegt zweifellos in der konsequenten Anwendung der Retention.

Es war wohl am 24. 1. 1896, daß er erstmals in der Wiener Ärztegesellschaft über seine in 25 Fällen seit 10 Monaten - also März 1895 - angewandte Methode sprach, allerdings ohne PAGI zu erwähnen.

Er gab dann 1896 am 25. Kongreß der Deutschen Gesellschaft für Chirurgie in Berlin seine Methode bekannt, und zwar auch hier ohne PACr zu erwähnen, worauf dann in der Diskussion Mikulicz hervorhob, «daß das Verfahren von Lorenz eng an das des italienischen Chirurgen PAGI anschließt», worin er zweifellos Recht hatte ${ }^{29}$.

Es folgte nun eine Zeit höchst unerfreulicher und unfeiner Polemiken, und zwar kämpfte hierin LoRenz gewissermaßen gegen zwei Fronten.

An der Spitze der einen Front stand Franz König, der Berliner Ordinarius für Chirurgie, der 1897 in der Berliner klinischen Wochenschrift einen scharfen Artikel gegen LoREnz publizierte: es sei begreiflich, bei der Luxation einen konservativen Versuch zu machen, es sei aber unwahrscheinlich, daß sich damit eine neue, tiefe Pfanne bilde. «Aber alle Erwägungen, welche darüber (sc. den Versuch) hinausgehen, sind zunächst unverstän-

29 Wir kennen übrigens im Balgrist eine Patientin (M.B.,H-No.9833, geb. 1891), bei welcher Lonenz 1896 links die Reposition durchführte und die er dann am Chirurgenkongreß demonstrierte. Die Patientin sagte mir, sie entsinne sich gut, wie damals ihre Eltern mit ihr nach Berlin zum Kongreß fuhren; sie wohnte damals in Dresden. Sie ist übrigens reluxiert mit einer schönen Sekundärpfanne. 
dig, sie gemahnen uns an das Verständnis des Kindes. ${ }^{30}$ Das war scharf und bitter und dazu wahrlich ungerechtfertigt. Und so ist es begreiflich, daß LoRenz in der gleichen Zeitschrift sich energisch gegen KöNIG wandte und ausführte, er wolle ja gar keine «neue Pfanne» bilden, sondern lediglich die vorhandene «umgestalten», und am Schluß seines Artikels heißt es: «... oder sollte die fruchtbare Mutter, Chirurgie" sich jetzt schon anschicken, ihre jüngste Tochter ,Orthopädie“ aus dem Hause zu verstoßen ?»

In der Tat war es so, daß diese unerfreulichen Zwistigkeiten mit dazu beitrugen, daß im September 1901 die Deutsche Orthopädische Gesellschaft gegründet wurde, die 1902 ihren ersten Kongreß abhielt.

Man kann somit sagen, daß Lorenz im Kampf gegen diese Front gesiegt hat.

Viel weniger erfreulich war die Polemik von Lonenz gegen PAci. Aber auch in dieser Polemik siegte Lorenz, aber nur scheinbar-eben deswegen, weil PACI bereits 1902 starb: les morts ont tort. In seiner Autobiographie Ich durfte helfen, in der LORENZ ausführlich von seinen Bemühungen und weltweiten Erfolgen spricht, wird PACI überhaupt nicht erwähnt. Auf eine Publikation PAcis, in welcher dieser seine Methode 1896 nochmals darlegte $^{31}$, antwortete LoRENZ in einem unerfreulich scharf gehaltenen "Offenen Brief ${ }^{32}$ : es gehe aus PAcIs Mitteilung hervor, «daß Ihnen eine veritable Einrenkung mit Ihrer Methode auch nicht ein einziges Mal geglückt ist», «Ihr palliativesVerfahren begnügt sich mit einer Stellungsverbesserung des Kopfes» und «Sie handeln in vollkommener Unkenntnis der Tatsachen » - das alles schrieb Lorenz, der doch in Rom PAcis Präparat gesehen und sich früher positiv über Pacıs Methode geäußert hatte!

Das alles ist sehr unerfreulich und wirft doch, bei allem Respekt, dem wir ihm zu zollen haben, ein etwas bedenkliches Licht auf Lorenz ${ }^{33}$.

30 Außerdem heißt es in Königs Artikel, es sei nicht Aufgabe, Ort und Zeit herzugeben in Sitzungen «zu ernster Facharbeit zusammenkommender Fachchirurgen ..., um als Organ zu dienen für jede Wendung, welche die Frage der Behandlung der Krankheit macht.»

31 A.PACI, Wiener klin. Wschr., Nr. 26, 1896.

32 A. Lorenz, Wiener klin. Wschr., Nr. 29, 1896.

33 PACIS Stil ist vornehmer und ruhiger als der Lonenzsche. Er schließt mit einem Schlußwort unter Hinweis auf sein Präparat die Diskussion 1896 und sagt: «Übrigens ist LoRENZ in Prioritätsstreitigkeiten kein Neuling mehr: zu der Zeit, da ich meine unblutige Methode schon lange anwandte, suchte sich Lorenz auf Grund einiger Modifikationen das Verdienst an der Erfindung der blutigen Methoden der angeborenen Hüftgelenks- 
Damit sollen ja nicht LoRENZ' ungeheuren Verdienste geschmälert werden. Hatte PACI einen wirklich brauchbaren Weg zur Reposition gewiesen, so hat Lorenz unter Übernahme der PAcischen Methode, unter Steigerung der PAcischen Hebelbewegungen vor allem durch die Einführung einer zweckmäßigen Retentionsmethode der geschlossenen Reposition Ende des 19. Jahrhunderts zum Siege verholfen. Trotzdem bleibt es richtiger, nicht von der Methode Lorenz zu sprechen, sondern von der Methode Paci Lorenz ${ }^{34}$.

Zum großen und verdienten Erfolg dieser Methode kam noch hinzu, daß sie gewissermaßen «im richtigen Moment » kam. Im gleichen Jahr (1895), in dem LoRenz die geschlossene Reposition aufbaute (nach über 200 offenen Repositionen) und immer mehr ausbaute, entdeckte C. Röntgen in Würzburg die nach ihm genannten Strahlen. Was das für Diagnose und Epikrise bedeutete, braucht nicht weiter ausgeführt zu werden ${ }^{35}$.

luxation beizulegen, welche ganz unzweifelhaft HoFFA gehörte. » - In diesem Zusammenhang sei erwähnt, daß es etwa 25 Jahre später hinsichtlich der Bifurkationsosteotomie ähnlich klang, als LoRENz 1920 schrieb: «Seit jeher an der Behandlung der irreponiblen Luxation lebhaft interessiert, hatten wir seit geraumer Zeit ganz genau denselben Heilplan entworfen und ausgeführt, als uns v.BAEYer durch seine Mitteilung zuvorkam.» Zur Beurteilung dieser Polemik geben folgende Sätze v. BaEYers Auskunft:

«1. Anfangs erkennt Lorenz mir die Priorität zu.

2. Dann beansprucht er sie für sich. Betont die Übereinstimmung unserer beiden Methoden.

3. Nach weiterem Verlauf behauptet er, daß unsere Methoden nicht dieselben seien.

4. Schließlich werde ich überhaupt nicht mehr erwähnt.»

${ }^{34}$ Zur Polemik Lorenz-Paci sagt Putti «Nessuno che sia a conoscenza delle fasi della disputa potrà tuttavia discognoscere che in essa il chirurgo italiano seppe sempre conservare una mirabile equanimità. Ma tutto ciò fa oggi mai parte della storia, la quale a quasiasi giudice spassionato presta bestevole argomento per distribuire il merito in giusta misura: ad Agostino Paci quello incontrovertibile di avere egli avuto per primo l'idea della cura incruenta e razionale della lussazione congenita dall'anca e di aver per primo insegnate le manovre di reduzione, ad Adolfo Lorenz quello certamente rispettabile di avere disciplinato la metodica della cura e di averne cosi accresciuta l'efficacia» (PUTtI, 1938).

${ }^{35}$ Die denkwürdige Sitzung, in welcher Röntgen seine Entdeckung bekanntgab, fand im Dezember 1895 in der Würzburger Physikalisch-medizinischen Gesellschaft statt.

Im gleichen Jahr wurde übrigens auch das Trendelenburgsche Phänomen beschrieben, das Lorenz diagnostisch 1905 als Lorenz-Trendelendurgschen Versuch bezeichnete, da er bereits früher "von Lorenz gewürdigt ... und später von Trendelenburg näher ausgeführt» worden war.

Daß in der Luxationstherapie aber sogar auch hier der Nationalismus sich bemerkbar machte, ersieht man daraus, daß Italiener von der «italienischen Methode» sprachen und LoRenz selbst 1924 seine Methode als «deutsche Methode» bezeichnete. 
Nach 1895 kam es zu einem «Sturm-und-Drang-Zeitalter unblutiger Reposition. Man glaubte das Universalmittel für jegliche Luxation jeglichen Alters gefunden zu haben » (Albert LoRenz, 1935). Man sprach von Dauerresultaten nach dreijähriger Beobachtung, und ich entsinne mich noch gut, mit welcher Ironie Scherb von GaUgele sprach, der von $100 \%$ igen Erfolgen berichtete.

Es braucht hier nicht auf all die nun auftauchenden Modifikationen dieser Methode eingegangen zu werden. Ihre Erfolge sind unbestritten, und wir haben Lorenz dankbar zu sein, daß er sie durchsetzte. Daß es Jahrzehnte ging, bis man ihre Grenzen und Gefahren erkannte ${ }^{36}$, erscheint begreiflich. Ihre Mißerfolge führten mit der Zeit dann doch dazu, daß die operative Behandlung wieder mehr zu ihrem Recht kam. Das weiter auszuführen, ist hier nicht mehr meine Aufgabe.

In einer Hinsicht erwies sich der Erfolg der Paci-Lorenz-Methode aber doch als nicht unbedenklich, ja als deletär. Wir denken hier an die Prophylaxe und an die Frühbehandlung. Wenn Lorenz noch 1920 schrieb: «Wir plädieren also dafür, die Behandlung erst dann zu beginnen, wenn die Luxation sich durch die Gehstörung zweifellos zu erkennen gibt. Im allgemeinen wird dies im Verlaufe des zweiten Lebensjahres der Fall sein ...», so wird doch der Eindruck erweckt, als habe hier die Autorität Lonenz' bremsend gewirkt.

Roser hatte schon 1879 Frühdiagnose und Frühbehandlung postuliert ${ }^{37}$. Aber erst etwa fünfzig Jahre später konnte, in erster Linie dank ViтToRio Putri, diese Behandlung Eingang finden und sich mehr und mehr durchsetzen $^{38}$.

Hinsichtlich der Prophylaxe der Luxation hatte bereits 1912 LE DAMANY, dem wir eine große Monographie verdanken, eine Bandage vorgeschlagen, die weitgehend der später, 1944, von PAVLIK angegebenen Bandage entsprach.

${ }^{36}$ Es sei hier nur an Fraenkels Ausspruch aus dem Jahre 1924 erinnert nach der Reposition bei einer 21jährigen Patientin: "Wir kennen heute für die unblutige Reposition keine Altersgrenze mehr.»!!

371953 publizierte B. DAvID dasselbe wie Roser als neue Methode, selbstverständlich ohne Roser zu nennen!

38 Paletta-Pravaz-Poggi-Paci-Putti: Es ist eigenartig, daß mit Ausnahme von Lorenz die Namen derer, die sich um die Luxation am meisten verdient gemacht haben, mit $\mathbf{P}$ anfangen! 
In der gleichen Epoche hat Froelich in seinem Wirkungskreis Nancy sich mit der Diagnose der Luxation vor Gehbeginn und vor allem mit deren Frühbehandlung befaßt; neben seiner eigenen Publikation ist hier vor allem auf die Arbeit seines Schülers J. E. P. Vuillaume zu verweisen (1911). Froelich, der die Spreizbehandlung mit einem dreieckigen Kissen seit 1910 anwandte und 1911 darüber am Kongreß der Société Française de Chirurgie sprach, konnte 1932 über 32 so geheilte Patienten berichten.

Aber das waren wohl Ausnahmen. Noch 1923 in seinen Prinzipien der Orthopädie schrieb P. Haglund: «Erst wenn das Kind zwei Jahre alt ist, kann eine erfolgversprechende Behandlung der angeborenen Hüftgelenksluxation begonnen werden ...»

Jedoch schon sechs Jahre später hat V. Putri über 24 Frühbehandlungen im ersten Lebensjahr berichtet und gab dann 1932 seine erste größere Statistik über 119 Frühbehandlungen seiner Therapie der prelussazione mit dem keilförmigen Spreizkissen bekannt, wobei der jüngste Patient 34 Tage alt war, die größte Gruppe aus dem vierten Monat stammte und diese Frühbehandlung bei Nachkontrollen sechs Mißerfolge aufwies. Wichtig war dann aber vor allem, daß Putri auf die Rolle der Mitarbeit der Geburtshelfer und vor allem der Pädiater in dieser prophylaktischen Frühbehandlung hinwies, die dann in Ferrara vor allem von Ortolani ausgebaut und propagiert wurde.

Damit sind wir mit der Frühbehandlung bzw. Prophylaxe bis fast in die Gegenwart gekommen, in welcher, neben den vorhin Genannten, vor allem Hilgenreiner, Freyka, F. Begker zu nennen sind.

Wenn ich 1959 einmal sagte, das Ziel der Sofort- und Frühbehandlung sei es, operative Eingriffe in der Behandlung der Luxatio coxae cong. so überflüssig zu machen, daß sie «hoffentlich später einmal nur noch für den Medizinhistoriker interessant sein werden», so mag das als ideales Ziel richtig sein. Wir sind aber leider noch lange nicht so weit. Noch lange, befürchte ich, werden wir übersehene Hüftdysplasien bzw. Hüftluxationen zu sehen bekommen (schon allein, weil es noch oft heißen wird: «das wächst sich aus!»), so daß offene Repositionen, varisierende DerotationsOsteotomien, Beckenosteotomien, hochdiaphysäre Osteotomien, um nur diese zu nennen, immer noch ihre Berechtigung haben werden. Ihre Besprechung gehört aber jetzt noch nicht in den Aufgabenkreis des Historikers.

Wir wollen aber nicht vergessen, daß es nunmehr fast 100 Jahre her sind, 1879, daß Roser auf Diagnose vor dem zweiten Lebensjahr drang und 
frühe Abduktionsbehandlung vorschlug und daß es noch keine $200 \mathrm{Jahre}$ her sind, daß Giovan Battista Paletta in seinem De claudicatione congenita 1783 die anatomischen Grundlagen für unsere Kenntnisse über die kongenitale Hüftluxation legte.

In diesen fast 200 Jahren ist ein langer Weg zurückgelegt worden. Das vor etwa 150 Jahren - DuPUYTREN - noch als unheilbar angesehene Leiden ist unter ganz bestimmten Bedingungen heilbar geworden. Der lange Weg war aber umsäumt von Hoffnungen und Enttäuschungen, von Freude und viel Leid, und auch an manch unerfreulichen Streitigkeiten hat es nicht gefehlt. Wenn ich aber an all die Luxationspatienten - und an ihre Angehörigen - denke, so kann ich nicht anders schließen als mit den Worten, die ich 1959 an einem Fortbildungskurs in Berlin sagte: «Die Literatur über die Luxatio coxae cong. ist unübersehbar. Ich kenne nur einen kleinen Teil von ihr. Ein Werk über sie fehlt aber. Es könnte den Titel tragen: «La grande pitié des luxations», oder auch: «Leidensgeschichten von Hüftgelenken ».

\section{Literatur}

ANdry N.,L'Orthopédie...,Paris 1741.

Bade Peter, Hippokrates und die angeborene Hüftverrenkung, Acta Chir.Scand.67 (1930) 35-41 (Festschrift Haglund).

- Die Geschichte der Deutschen Orthopädischen Gesellschaft, Ebering, Berlin 1939.

Baeyer H.von, Die Bifurkation nach Baeyer-Lorenz. Ein Protest. Z.orthop.Chir. 44 (1924) 591-593.

Bick Edgar M., Source Book of Orthopaedics, Williams and Wilkins Comp., Baltimore 1948.

Borst Max, Die cong. Hüftgelenksluxation, Med.Diss., Würzburg 1892.

Castiglioni A., Histoire de la médecine, Payot, Paris 1931.

Chledowski C.von, Der Hof von Ferrara, Müller, München 1919.

David B.,Zur Behandlung der flach-dysplastischen Hüftpfanne durch beidseitigen Unterschenkelgips mit abduzierenden Stab. (1953) Z. Orthop. 83 319-321.

Delpech J.M.,L'Orthomorphie, Deutsche Ausgabe, Weimar 1930.

Dupurtren G., Mémoire sur un déplacement originel ou congénital de la tête des fémurs. Rep.gen.Anat.Physio.Acad.Méd.Paris, 1826 (zit.nach Delpech und nach Pravaz).

Fischer Eugen, Heinrichs des Löwen sterbliche Reste, Die Welt als Geschichte 12 (1952) 233-241.

- Angeborene oder traumatische Hüftgelenksluxation an den Herzog Heinrich dem Löwen zugeschriebenen Gebeinen, Arch.orthop. Unf. Chir.48 (1956) 352-366.

Francillon M.R., Zur operativen Therapie der Dysplasia luxans coxae, Med.Welt (1960) $17-23$. 
Froelich, Zur Prophylaxe der angeborenen Hüftverrenkung, Z. Orthop. 56 (1932) 587-591.

Gabory Emile, Anne de Bretagne, Duchesse et Reine, Plon, Paris 1941.

GAUGELE K., Über die Behandlung der angeborenen Hüftgelenks-Verrenkung, Z.orthop. Chir. 34. (1914) 441-478.

Gurut E., Geschichte der Chirurgie, Band I, Hirschwald, Berlin 1898.

Hackenbroch M. und W. Holtzmann, Die angeblichen Überreste Heinrichs des Löwen, Dtsch.Arch.Erforsch.Mittelalter (Mon.Germ.Hist.) 10 (1954) 488-503.

Haglund P.,Die Prinzipien der Orthopädie, Fischer, Jena 1923.

Hengen Otto P., Paläomedizin Altägyptens, Roche Image 45 (1971) 25-33.

Hoffa A., Über die operative Behandlung der angeborenen Hüftgelenksluxation, Atti XI ${ }^{o}$ Congresso Med.internat.Roma, Vol.IV,360,29. 3. fino 5.4.1894, Torino 1895.

Jones A.R., Cong.Dislocation of the Hip.Modern Trends in Orthopaedics, Butterworth, London 1950.

Kirmisson E., Contribution à l'étude de la pathogénie et du traitement des luxations congénitales de la hanche, Atti XI ${ }^{\circ}$ Congresso Med.internat.Roma, Vol.IV, 361-378, 29.3. fino 5.4.1894, Torino 1895.

KöNig F., Die cong. Luxation des Hüftgelenkes, Berliner klin.Wschr., Nr.2 (1897) 21-22.

Lacroix PaUl, Louis XII et Anne de Bretagne, Paris 1882.

Le Damany P., La Luxation cong. de la hanche, Alcan, Paris 1912.

Leveuf J. et P.Bertrand, Luxations et Subluxations cong. de la Hanche, Doin, Paris 194.6.

Lorenz Adolf, Pathologie und Therapie der angeb. Hüftverrenkung auf Grundlage von 100 operativ behandelten Fällen, Urban \& Schwarzenberg, Wien/Leipzig 1895.

- Die operative Therapie der Luxatio coxae congenita. Atti XI ${ }^{o}$ Congresso Medico internat. Roma, Vol.IV, 357-360,29.3. fino 5.4.1894, Torino 1895.

- Zur Priorität der unblutigen Reposition der angeborenen Hüftverrenkung (Offener Brief an Herrn Agostino Paci), Wiener klin. Wschr., Nr. 29 (1896) 658-660.

- Zur cong. Luxation des Hüftgelenks, Berliner klin.Wschr., Nr.6 (1897) 112-114.

- Über die unblutig-chir. Behandlung der angeborenen Hüftverrenkung mittels der funkt. Belastungsmethode, Volkmann, Sammlung Klin.Vorträge, Nr.151/152,1896.

Lorenz AD. und M.Reiner, Die angeborene Hüftverrenkung, Joachimsthal Hdb.orthop. Chir.II, 2 (1905-1907) 65-260.

LoRenz Adolf, Die sogenannte angeborene Hüftverrenkung, Enke, Stuttgart 1920.

- Autobiographie, Die Medizin in Selbstdarstellungen, Band III, Meiner, Leipzig 1924.

- Ich durfte helfen, Staakmann, Leipzig 1937.

Lorenz Albert, Der gegenwärtige Stand der Hüftluxations-Therapie, Z. orthop. Chir.63 (1935) 93-128.

Mondor H., Anatomistes et Chirurgiens, Fragrance, Paris 1949.

Nicholson J.T., Founders of American Orthopaedics, J.Bone Joint Surg. 48 A.(1966) 582-597.

Ortolani M.,La Lussazione cong. dell'anca, Capelli, Bologna 1948.

$\mathrm{P}_{\mathrm{ACI}}$ A., Della lussazione iliaca comune congenita del femore, Atti $\mathrm{XI}^{\circ}$ Congresso Med. internat. Roma. Vol. IV, 378-411,29.3. fino 5.4.1894, Torino 1895.

- Zur Lorenz'schen Methode der unblutigen Behandlung der angeborenen Hüftgelenksluxation, Wiener klin. Wschr., Nr.26 (1896) 567. 
- Schlußwort zu meiner Diskussion mit Herrn Professor Lorenz, Wiener klin. Wschr., Nr. 33 (1896) 756.

Paletta G. B., Adversaria chir., Milano 1783 (zit. nach Delpech und nach Pravaz).

- Exercitationes pathologicae, 1820 (zit. nach Pravaz).

Poggi A., Contributo alla cura cruenta della lussazione cong. dell'anca, Arch.ortop.7 (1888) 105 (zit.nach Lorenz 1895 und 1920).

Pravaz Ch. G., Traîté théorique et pratique des Luxations congénitales du Fémur, Baillère, Paris 1847.

Putri V., Statistica degli stati di prelussazione cong.dell'anca curati col metodo dell'abduzione, Chir.Org. Mov.17 (1932) 209-217.

- Giovan Battista Paletta, Chir.Org. Mov. 23 (1937) 105-107.

- Agostino Paci, Chir. Org. Mov. 23 (1938) 307-309.

- Fedele Margary, Chir.Org. Mov.23 (1938) 395-398.

RöNTGen W.C., Über eine neue Art von Strahlen, Sitzungsber. Würzburger Phys.-med. Ges. 1895, in H.R.Schinz, 60 Jahre medizinische Radiologie, Thieme, Stuttgart 1959.

Roser W., Über angeborene Hüftverrenkung, 8. Kongreß Dtsch.Ges.Chir.17.4.79, Arch. klin. Chir.24 (1879) 309-313.

SAнiв Pн., Orthop. and Traumatic Skeletal Lesions in Ancient Egyptians, J.Bone Joint Surg.44 B. (1962) 944-947.

Sснмірт Rich., Heinrich der Löwe, seine Stellung in der inneren und auswärtigen Politik Deutschlands, Hist.Z.154 (1936) 241-284.

Stromeyer G.F.L., Handbuch der Chirurgie, Herder, Freiburg i. Br. 1844/64.

Trendelenburg F., Über den Gang bei angeborenen Hüftluxationen, Dtsch.med. Wschr. 91 (1895).

Valentin B., Geschichte der Orthopädie, Thieme, Stuttgart 1961

Vuillaume J.E.P., La lux.cong. de la hanche chez l'enfant qui n'a pas encore marché, Med. Diss., Nancy 1911.

Wells Calvin, Hip Disease in ancient man, J.Bone Joint Surg. 45 B. (1963) 790-791.

Würtz Hans, Zerbrecht die Krücken, Voss, Leipzig 1932. 